\title{
Globalization of MIGS
}

\author{
Chelvin C. A. Sng, Clement C. Tham, Donald L. Budenz, \\ Paul R. Healey, and Ningli Wang
}

\subsection{Introduction}

Good health for all populations is an accepted international goal. Globalization is the process of interaction and integration among people, companies, and governments worldwide and was previously regarded as a predominantly economic process. However, it is increasingly perceived as a more comprehensive phenomenon with significant implications for global health. The relationship between globalization and health is complex and globalization is a multifaceted phenomenon which can influence health in a myriad of ways. Globalization facilitates the spread of modern medicine and medical devices, extending life expectancy in developing countries from 55 years in 1970 to 65 years in 1997. However, it can also exacerbate the gap between the rich and the poor, both among and within countries.

\section{C. A. Sng $(\bowtie)$}

Department of Ophthalmology, National University Hospital, Singapore, Singapore

Singapore Eye Research Institute, Singapore, Singapore

C. C. Tham

Department of Ophthalmology and Visual Sciences, The Chinese University of Hong Kong, Shatin, New Territories, Hong Kong SAR

Hong Kong Eye Hospital, Kowloon, Hong Kong SAR

D. L. Budenz

Department of Ophthalmology, School of Medicine, University of North Carolina at Chapel Hill, Chapel Hill, NC, USA

P. R. Healey

Centre for Vision Research, Westmead Institute for Medical Research, University of Sydney, Sydney, NSW, Australia

N. Wang

Beijing Tongren Eye Center, Beijing Tongren Hospital, Capital Medical University, Beijing Ophthalmology and Visual Science Key Laboratory, Beijing, China 
While knowledge of and interest in minimally invasive glaucoma surgery (MIGS) has spread across the globe, access is far from global. Prior to 2016, MIGS devices were available mainly in Europe and Canada, with the exception of the iStent trabecular micro-bypass stent, which was also commercialized in the United States and selected countries in Asia (Singapore and Hong Kong). Since 2016, the US Food and Drug Administration (FDA) has approved the CyPass Micro-Stent (Alcon Laboratories Inc., Fort Worth, Tx USA) (August 2016, subsequently withdrawn in August 2018), the XEN Gel Implant (Allergan plc, Dublin, Ireland) (November 2016), the iStent Inject (Glaukos Corporation, San Clemente, CA, USA) (June 2018), and the Hydrus Microstent (Ivantis Inc., Irvine, CA, USA) (August 2018). These devices are also slowly making inroads into Asia, Australasia, South America, and South Africa.

The global prevalence of glaucoma is anticipated to increase from 64.3 million in 2013 to 111.8 million in 2040, disproportionately affecting people in Asia and Africa [1]. MIGS companies may foresee a quantum leap in economies of scale by serving global markets rather than only a confined domestic market.

\subsection{Cost}

In view of the costly commercialization process and the expensive acquisitions of several MIGS start-ups by multinational companies, MIGS devices are currently priced at a premium. For instance, the price of the XEN Gel Implant has more than doubled in Europe since Aquesys was acquired by Allergan in 2015. Hence, the goto-market strategy for most MIGS companies has focused on countries where a significant proportion of healthcare coverage is provided through private health insurance (e.g., United States and Australia) or public health funding (e.g., the United Kingdom and Canada). The latter often requires the technology to be appraised and recommended by certain national institutes based on clinical and economic evidence. For instance, the National Institute for Health and Care Excellence (NICE) in the United Kingdom carries out periodic health technology assessments of new entrants. While not legally binding, a NICE assessment carries a great deal of weight for or against the adoption of new technology.

Some studies have reported MIGS cost-effectiveness in such reimbursement environments. In the Canadian healthcare setting, Patel et al. projected that two iStent Trabecular Micro-Bypass Stents were cost-effective compared with standardof-care treatment with glaucoma medication in patients with mild-to-moderate open-angle glaucoma for more than 15 years, with quality-of-life gains [2]. Ngan et al. compared iStent accompanying cataract surgery with topical glaucoma medication in a public healthcare setting in New Zealand and found that the iStent is reasonably cost-effective, particularly for those using more expensive topical glaucoma medications [3]. Indeed, the cost-effectiveness of MIGS procedures may vary depending on whether brand name or generic eye drops are used. In the Manchester iStent study, Tan and Au found that the overall cost of combined cataract surgery and iStent implantation was GBP£7.70 per patient per year more than conservative management with brand name eyedrops, but GBP£131.3 per patient per year more 
if generic eyedrops were used [4]. Nevertheless, a systematic literature review of clinical and economic outcomes of MIGS in primary open-angle glaucoma highlighted that the available evidence on the cost-effectiveness of MIGS is limited and it remains unclear whether the cost of using MIGS is outweighed by cost savings through decreased medication and need for further interventions [5]. The price-performance ratio of MIGS is also an important consideration. The Microcatheter (iTrack 250A, iScience Interventional, Menlo Park, CA, USA) and Trabectome (NeoMedix Corporation, San Juan Capistrano, CA, USA) are available in the Chinese market, but these procedures are expensive and the surgical expenses are not covered by basic medical insurance. Hence, they are not widely performed in China. Evidently, there is still significant room for growth in the large Chinese glaucoma surgical market.

Ultimately, the global scalability of MIGS would be more dependent on cost and ease of implantation than efficacy. Unfortunately, out-of-pocket payments constitute a significant part of the health financing landscape in many countries. While MIGS may still be affordable in the wealthier of such countries (e.g., Singapore and Hong Kong), the cost of these devices is prohibitive for a significant proportion of the world's population, especially in Asia and Africa. In these large albeit costsensitive markets, the cheapest MIGS device is likely to prevail and outperform its competitors. In particular, current treatment options for glaucoma patients in developing countries remain limited and unsustainable. Even if these patients are diagnosed at an early stage of the disease, their prognosis remains dire. The recurrent cost of glaucoma medication renders them unaffordable in the long run. The lack of patient understanding of their disease results in poor compliance with medication and conventional glaucoma surgery (i.e., trabeculectomy) is often not feasible due to the difficulty of postoperative care, unpredictable results, and possible surgical complications. New glaucoma surgical treatment options, for example MIGS, that are safer and require less postoperative management, are particularly promising in this context. Ordonez et al. projected that the iStent Trabecular Micro-Bypass Stent would be a highly cost-saving procedure in the Colombian healthcare system due to more quality-adjusted life-years related to a lower rate of the population with loss of visual acuity in the long term [6]. Despite being potentially vision-saving, the cost of these new innovations prohibits their use in developing countries where they are most needed and the potential surgical volumes are the highest. Reducing the price of these devices in exchange for an increase in sales volume will chart a more profitable path in these resource-poor markets.

\subsection{Distribution Channels}

After Glaukos Corporation, which developed the iStent Trabecular Micro-Bypass Stent, staged a hugely successful initial public offering (IPO) in June 2015, the MIGS world has been rocked by a series of high-profile and multimillion dollar acquisitions. Allergan purchased Aquesys and its XEN Gel Implant with a US\$325 million upfront payment in the third quarter of 2015. This was followed by the similarly expensive acquisitions of Transcend Medical and its CyPass Micro-Stent by 
Alcon (withdrawn in August 2018) and the InnFocus MicroShunt (now renamed PRESERFLO MicroShunt) by Santen in 2016. With these developments, the MIGS landscape has evolved rapidly from a medley of start-ups to a battleground of industry heavyweights.

The acquisition of MIGS devices by multinational pharmaceutical and biotechnology companies, which have established international distribution channels, will undeniably facilitate the globalization of MIGS. In contrast, the smaller MIGS companies that have not been acquired often have to rely on local distributors to access international markets and have to be more selective with their target markets. There are certainly opportunities for mutually beneficial partnerships to be forged between the MIGS companies in their quest for global expansion. An illustrative example is a multi-year agreement between Glaukos and Santen whereby Glaukos will become the exclusive distributor of the MicroShunt solely in the US market once it is approved by the FDA. This will allow Santen to concentrate on the European and Asian markets while leveraging on Glaukos's established distribution and sales infrastructure in the United States.

\subsection{Surgical Training}

A major constraint in the globalization of MIGS is the limited resources available for surgeon training and education. The inadequate availability of surgical trainers (who may require years of experience before reaching competence) and real patients for supervised surgery creates a bottleneck in the training of MIGS surgeons. In addition, the number of free training devices provided by MIGS manufacturers is often insufficient for surgeons to overcome their learning curve. To address these deficiencies and to prevent them from compromising training standards, an expanded role for augmented and virtual reality in MIGS surgical training should be explored [7]. Virtual reality-based simulators for MIGS surgery may be able to reduce the learning curve, improve the conceptual understanding of ocular anatomy and enhance visuospatial skills, augmenting (albeit not replacing) the guidance provided by surgical trainers. Improvements in information technology have also dramatically increased the speed and ease of data flow, facilitating the sharing of information. To rapidly disseminate didactic training material on MIGS, such resources should be made freely available online in various languages. However, surgeons should be advised to access such information only through reputable websites and sources, so as to prevent the potentially rapid dissemination of misinformation. For instance, www.migs.org is a noncommercial site offering patient information leaflets in multiple languages. The Asia-Pacific Glaucoma Society has established a MIGS Interest Group and developed patient information leaflets for MIGS devices which are available online (www.apglaucomasociety.org/migs) (Fig. 11.1). Other international and regional glaucoma societies may consider similar initiatives to improve surgeon and patient education. It is imperative that surgical standards are not compromised in the process of globalization, to ensure consistently good outcomes from MIGS. 


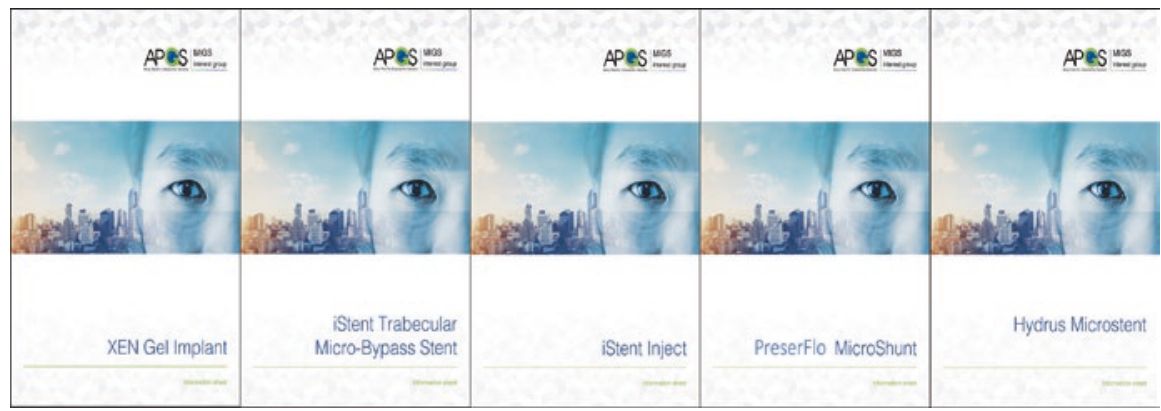

Fig. 11.1 Asia-Pacific Glaucoma Society patient information leaflets for minimally invasive glaucoma surgery devices which are available at www.apglaucomasociety.org/migs. (Image provided by Chelvin Sng, FRCSEd)

Fig. 11.2 Anterior segment photograph of a diffuse bleb associated with the XEN Gel Implant in a Chinese eye with thick Tenon's capsule, showing that the implant is not visible when it is located under the Tenon's capsule. (Copyright Chelvin Sng, FRCSEd; reproduced with permission)

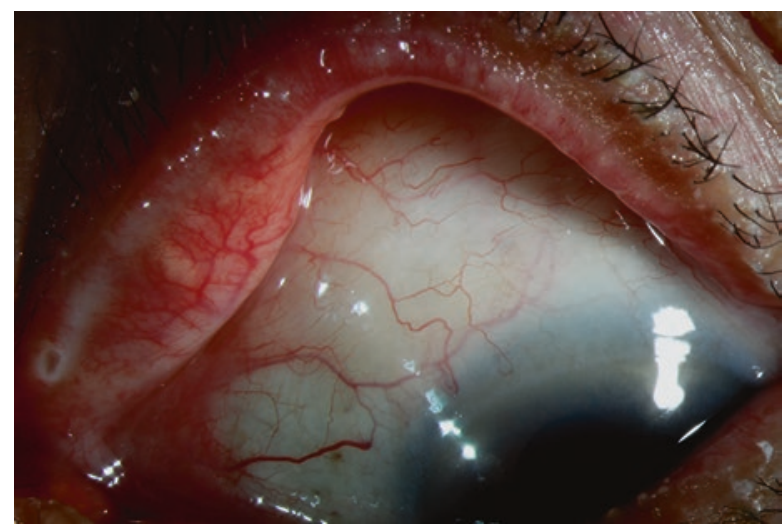

\subsection{Patients}

Currently, the published data on MIGS procedures mostly pertain to Caucasian eyes and it remains uncertain whether these devices will be just as effective in Asian or African eyes. This is especially relevant for the subconjunctival MIGS devices, as Asian and African eyes have a propensity for subconjunctival scarring [8, 9]. The Tenon's capsule is also significantly thicker in African and Asian eyes compared with Caucasian eyes, which may predispose to the occlusion of subconjunctival MIGS devices. For instance, the XEN Gel Implant is an ab-interno subconjunctival MIGS device that is typically implanted without a conjunctival peritomy, but in some African and Asian eyes with very thick Tenon's (Fig. 11.2), it may be necessary to perform a conjunctival peritomy to ensure proper placement of the implant (Fig. 11.3). It may also be necessary to apply a higher concentration of antimetabolites intraoperatively and to prescribe a longer duration of postoperative topical 
Fig. 11.3 Implantation of the XEN Gel Implant with conjunctival peritomy. (Copyright Chelvin Sng, FRCSEd; reproduced with permission)

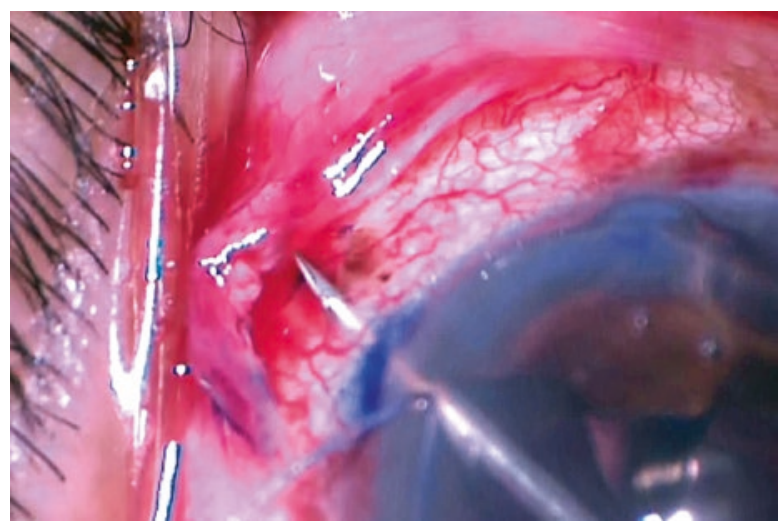

steroids for subconjunctival MIGS devices to achieve similar rates of surgical success as that in Caucasian eyes, although further studies are required to verify these hypotheses.

Despite the size of the Chinese market, with a high prevalence of glaucoma, the availability of MIGS and other novel glaucoma surgical options is currently very limited. One exception is the Microcatheter (iTrack 250A, iScience Interventional, Menlo Park, CA, USA), which was introduced in China in 2012 by Wang's team. This typically requires a conjunctival peritomy and the creation of a scleral flap and is not considered minimally invasive. Wang's team subsequently simplified the canaloplasty surgical procedure in China to introduce reconstruction of aqueous outflow drainage (RAOD) surgery, omitting two steps (creation of the scleral lake and Descemet's membrane window). This simplified procedure has been used to treat eyes with multiple failed glaucoma filtration procedures and disrupted Schlemm's canal observed by gonioscopy (relay technique) [10]. They also pioneered the use of partial circumferential trabeculotomy with the Microcatheter in primary congenital glaucoma after failed angle surgeries, which may achieve similar postoperative IOP as $360^{\circ}$ trabeculotomy. Refractory glaucoma with previous failed conventional glaucoma filtration procedures is a significant challenge in China and bleb-less surgery may be a viable surgical option for these eyes, although the postoperative IOP may not be low enough for advanced glaucoma. The Trabectome is also available in China and has been shown to be effective and safe in a multicenter retrospective study [11]. With the introduction of such new surgical options, trabeculectomy rates in China have gradually declined. The surgical outcomes of other MIGS procedures in Chinese eyes still require investigation.

Data on MIGS outcomes in glaucoma subtypes besides primary open-angle glaucoma are limited. The efficacy of MIGS in certain subtypes of glaucoma, such as angle-closure glaucoma which is prevalent in Asia, would need to be established. In an exploratory, prospective, interventional case series, Hernstadt et al. showed that combined iStent trabecular micro-bypass stent insertion and phacoemulsification was effective in lowering the intraocular pressure $(14.8 \pm 3.9 \mathrm{vs} .17 .5 \pm 3.8 \mathrm{mmHg}$ at baseline; $p=0.008)$ and the number of glaucoma medications $(0.14 \pm 0.48$ vs. 
$1.49 \pm 0.77$ at baseline; $p<0.001$ ) for at least 12 months, with a favorable safety profile. Despite cataract extraction, postoperative iStent occlusion with iris occurred in $27.0 \%$ of the eyes, which is much higher than the incidence of stent occlusion reported after iStent implantation in eyes with open-angle glaucoma (4-18\%) [12]. In a prospective, single-masked, randomized study comparing the efficacy of combined phacoemulsification and iStent implantation with standard phacoemulsification in eyes with primary angle closure disease, the combined surgery was associated with a higher likelihood of complete success at 12 months $(87.5 \%$ [95\% CI 58.6-96.7 vs 43.8\% [95\% CI 19.8-65.6]; $p=0.01$ ) [13]. Sng et al. reported that combined XEN implantation with cataract surgery was effective in lowering IOP and the number of glaucoma medications in 31 Chinese eyes with primary openangle glaucoma and primary angle-closure glaucoma for at least 12 months, with a favorable safety profile [14]. However, implant occlusion with iris may occur more frequently in angle-closure eyes, even after cataract surgery (Fig. 11.4). This exploratory study was unable to distinguish between the IOP-lowering effect of XEN and phacoemulsification and more randomized controlled studies comparing combined phacoemulsification with MIGS and phacoemulsification alone are required.

Trabecular bypass procedures and newer modalities of cycloablation are attractive surgical options for patients in less developed nations as they do not require much postoperative management or medications. However, cycloablative procedures may increase conjunctival scarring and compromise the success of subsequent
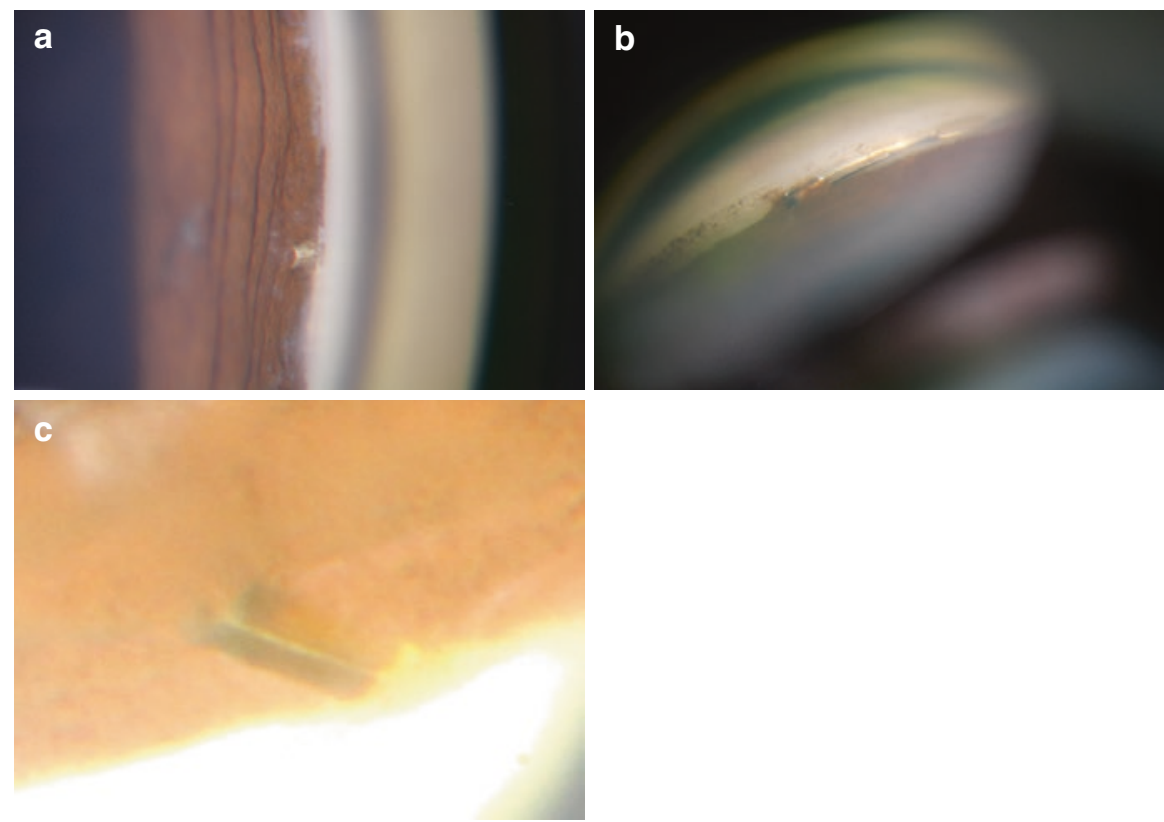

Fig. 11.4 Gonioscopy photographs showing iris occlusion of the (a) iStent Trabecular MicroBypass Stent, the (b) Hydrus Microstent, and the (c) XEN Gel Implant in angle-closure glaucoma eyes even after cataract extraction. (Copyright Chelvin Sng, FRCSEd; reproduced with permission) 
conjunctival filtration surgery [15]. Trabecular bypass procedures have a high safety profile and spare the conjunctiva, but the modest efficacy of these procedures renders them more appropriate for patients with the mild-to-moderate disease who would otherwise be noncompliant with their glaucoma medications. In reality, due to the poor access to healthcare and eye screening programs, a significant number of patients in less developed nations present with advanced glaucoma. Subconjunctival MIGS devices, such as the InnFocus Microshunt (renamed PRESERFLO MicroShunt), are more likely to achieve lower target IOP compared with other MIGS devices, with a lower rate of complications compared with trabeculectomy [16], but require postoperative bleb management and possibly a longer duration of topical steroids. There is currently an unmet need for a MIGS device that requires minimal postoperative management and yet is able to reduce IOP sufficiently for patients with advanced glaucoma.

\subsection{Regulatory Considerations and Other Concerns}

Regulatory restrictions remain a roadblock for the globalization of MIGS. It is important for MIGS manufacturers to understand the legal and regulatory climate before entering a new market. Such information is vital for strategic decisions about where and how to expand globally. Countries with fewer regulatory barriers (e.g., Europe, Canada, and Singapore) often have earlier access to MIGS devices. On the other hand, certain countries (e.g., United States and China) have more stringent requirements. The US-FDA's strict adherence to evidence-based evaluation of medical devices often results in a notoriously drawn-out and bureaucratic approval process. The Chinese FDA (CFDA) requirement for data from Chinese patients is similarly challenging. When submitting international multicenter clinical trial data for CFDA evaluation, the overseas applicant is obliged to show that the enrolled Chinese trial subjects are representative of the relevant patient population in Chinese medical practice and that the sample size of Chinese subjects meets statistical requirements. As it may be daunting to conduct a clinical trial in China, a feasible alternative would be to obtain supplementary data from other countries with predominantly Chinese populations (e.g., Singapore and Hong Kong). Nevertheless, the United States and China are two of the largest markets globally and remain coveted despite their regulatory difficulties. While most MIGS manufacturers target the US market after establishing their devices in Europe, a notable exception is EyeTechCare (manufacturer of High-Intensity Focused Ultrasound [EyeOP1 HIFU, EyeTechCare, Rillieux-la-Pape, France]), which received CFDA approval for its device ahead of US-FDA approval. This alternative commercialization strategy affords the company the first-mover advantage in a large and untapped Chinese market, although the long-term advantages of this strategy are yet to be established.

Another consideration in the globalization of MIGS is the protection of intellectual property, which remains a concern in lesser-developed countries despite the Agreement on Trade-Related Aspects of Intellectual Property Rights (1994). Indeed, 
it may be difficult to seek legal recourse for intellectual property infringements in certain countries, and under such circumstances, the value of novel biomaterials or trade secrets may exceed that of patents in intellectual property protection.

\subsection{Conclusion}

The path to MIGS globalization is fraught with adversity as well as opportunity. Despite the economic, logistic, training, legal and regulatory challenges discussed earlier, the permeation of MIGS devices internationally is an inevitable reality. Ultimately, the success of these devices in the global market will distinguish the survivors from the casualties. It is anticipated that the health benefits of globalization will outweigh the disadvantages, and glaucoma patients worldwide stand to gain from increased access to MIGS devices, which might reduce glaucoma medication burden and improve quality of life. Whether such new technology can decrease the global incidence of glaucoma-related blindness remains to be seen, but this is certainly a worthy aspiration.

\section{References}

1. Quigley HA. Number of people with glaucoma worldwide. Br J Ophthalmol. 1996;80:389-93.

2. Patel V, Ahmed I, Podbielski D, Falvey H, Murray J, Goeree R. Cost-effectiveness analysis of standalone trabecular micro-bypass stents in patients with mild-to-moderate open-angle glaucoma in Canada. J Med Econ. 2019;22:390-401.

3. Ngan K, Fraser E, Buller S, Buller A. A cost minimisation analysis comparing iStent accompanying cataract surgery and selective laser trabeculoplasty versus topical glaucoma medications in a public healthcare setting in New Zealand. Graefes Arch Clin Exp Ophthalmol. 2018;256:2181-9.

4. Tan SZ, Au L. Manchester iStent study: 3-year results and cost analysis. Eye (Lond). 2016;30:1365-70.

5. Agrawal P, Bradshaw SE. Systematic literature review of clinical and economic outcomes of micro-invasive glaucoma surgery (MIGS) in primary open-angle glaucoma. Ophthalmol Ther. 2018;7:749-73.

6. Ordonez JE, Ordonez A, Osorio UM. Cost-effectiveness analysis of iStent trabecular microbypass stent for patients with open-angle glaucoma in Colombia. Curr Med Res Opin. 2019;35:329-40.

7. Jacobsen MF, Konge L, Bach-Holm D, et al. Correlation of virtual reality performance with real-life cataract surgery performance. J Cataract Refract Surg. 2019;45:1246-51. [Epub ahead of print]

8. Nguyen AH, Fatehi N, Romero P, et al. Observational outcomes of initial trabeculectomy with mitomycin $\mathrm{C}$ in patients of African descent vs patients of European descent: five-year results. JAMA Ophthalmol. 2018;136:1106-13.

9. Husain R, Clarke JC, Seah SK, Khaw PT. A review of trabeculectomy in East Asian peoplethe influence of race. Eye (Lond). 2005;19:243-52.

10. Xin C, Tian N, Li M, Wang H, Wang N. Mechanism of the reconstruction of aqueous outflow drainage. Sci China Life Sci. 2018;61:534-40. 
11. Dang YL, Cen YJ, Hong Y, et al. Safety and efficacy of trabectome-mediated trabecular meshwork ablation for Chinese glaucoma patients: a two-year, retrospective, multicentre study. Chin Med J. 2018;131:420-5.

12. Hernstadt DJ, Cheng J, Htoon HM, Sangtam T, Thomas A, Sng CCA. Case series of combined iStent implantation and phacoemulsification in eyes with primary angle closure disease: oneyear outcomes. Adv Ther. 2019;36:976-86.

13. David Z, Chen, Chelvin CA. Sng, Tiakumzuk Sangtam, Anoop Thomas, Liang Shen, Philemon K. Huang, Jason Cheng. Phacoemulsification vs phacoemulsification with micro-bypass stent implantation in primary angle closure and primary angle closure glaucoma: A randomized single-masked clinical study. Clin Exp Ophthalmol. 2020;48:450-61.

14. Chelvin C. A. Sng, Paul T. K. Chew, Hla Myint Htoon, Katherine Lun, Preethi Jeyabal, Marcus Ang. Case Series of Combined XEN Implantation and Phacoemulsification in Chinese Eyes: One-Year Outcomes. Adv Ther. 2019;36:3519-529.

15. Nicholas Y. Q. Tan, Marcus Ang, Anita S. Y. Chan, Veluchamy A. Barathi, Clement C. Tham, Keith Barton, Chelvin C. A. Sng. Transscleral cyclophotocoagulation and its histological effects on the conjunctiva. Sci Rep. 2019;9.

16. Santen Pharmaceutical. Santen Announces Topline Data for DE-128 (MicroShunt) Demonstrating Reductions in IOP and Medication Use in Patients with Glaucoma. Press Release, 30 Aug 2019. https://eyewire.news/articles/santen-announces-topline-data-for-de128-microshunt-demonstrating-reductions-in-iop-and-medication-use-in-patients-with-glaucoma/. Accessed 18 Sep 2019.

Open Access This chapter is licensed under the terms of the Creative Commons Attribution 4.0 International License (http://creativecommons.org/licenses/by/4.0/), which permits use, sharing, adaptation, distribution and reproduction in any medium or format, as long as you give appropriate credit to the original author(s) and the source, provide a link to the Creative Commons license and indicate if changes were made.

The images or other third party material in this chapter are included in the chapter's Creative Commons license, unless indicated otherwise in a credit line to the material. If material is not included in the chapter's Creative Commons license and your intended use is not permitted by statutory regulation or exceeds the permitted use, you will need to obtain permission directly from the copyright holder.

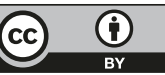

